\title{
Signal-centered action patterns in rats without neocortex in a Pavlovian conditioning situation
}

\author{
DAVID A. OAKLEY, LESLEY C. EAMES, and JANET L. JACOBS \\ Department of Psychology and Centre for Neuroscience, University College London \\ London WCIE 6BT, England
}

\author{
and \\ GRAHAM C. L. DAVEY and GARY C. CLELAND \\ Department of Social Science and Humanities, The City University, London ECIV OHB, England
}

\begin{abstract}
After removal of neocortex, rats were found to press a lever more readily than were shamoperated controls when exposed to an autoshaping procedure in which either the movement of a lever (Experiment 1) or its illumination (Experiment 2) was paired with the delivery of food. In both experiments, the apparatus was provided with a second, but nonpredictive, lever. As in more traditional Pavlovian situations, the neodecorticates showed clearer-thannormal response differentiation between the two cues, as well as more complete reversal of responding when their predictiveness was altered. Despite abnormally strong acquisition of autoshaped responding, the lesioned animals were able to suppress these responses under an omission schedule (Experiment 3). These data make it unlikely that the instrumental leverpress deficit seen in neodecorticated rats and rabbits in earlier studies is due in any simple way to sensory, motor, or motivational limitations in the lesioned animals and suggest a cause elsewhere in the learning paradigm itself.
\end{abstract}

The emergence of action patterns more usually associated with instrumental learning under a Pavlovian regime of stimulus presentation in the autoshaping situation (Boakes, 1977; Hearst \& Jenkins, 1974; Jenkins, Barrera, Ireland, \& Woodside, 1978) makes it a potentially powerful tool in an investigation of the neural substrates of Pavlovian conditioning and instrumental learning (Oakley, 1979a, 1979d; Russell, 1966, 1971). Rats and rabbits with all neocortex surgically removed (neodecorticated) have been shown to learn within normal limits when Pavlovian conditioning procedures are used (DiCara, Braun, \& Pappas, 1970; Oakley \& Russell, 1972, 1974, 1975, 1976, 1977), and are similarly unimpaired as far as simple instrumental responses, such as running an alleyway for food, are concerned (Oakley, 1979c, 1981). When neodecorticates are faced with an instrumental free-operant situation that requires them to press a lever or treadle for food reward, however, they are slow to acquire the response and are grossly inefficient when the response must be repeated several

This work was supported in part by Grants GR/A 56117 and GR/B 19321 from the Science Research Council of Great Britain. G.C.C. is in receipt of a Wellcome Trust Scholarship. The authors are grateful to $C$. Cromarty for help in preparing the illustrations. Address correspondence to David A. Oakley, Department of Psychology, University College London, Gower Street, London WC1E 6BT, England. times for each reward (Oakley, 1971; Oakley \& Russell, $1978 \mathrm{a}, 1978 \mathrm{~b}, 1979,1980)$ unless they are specifically pretrained (Oakley, 1979b, 1980). It could be that free-operant situations of this sort require the formation of response-reward associations that are more complex than those required for alleyway running and that are beyond the learning capacities of the neodecorticate. Alternatively, the fact that the freeoperant deficit can be overcome by pretraining designed to draw the animal's attention to the manipulandum suggests that the neodecorticate's deficit may not be an associative one but one of paying sustained attention to, or identifying, the manipulandum (Oakley, 1979a, 1979b, 1979d, 1980; Oakley \& Russell, 1978a, 1980). There is, however, a more fundamental explanation than either of these for the difference seen in neodecorticates in the two types of instrumental situation mentioned. In the absence of its cortical sensory and motor areas, the neodecorticate may simply have difficulty in locating and operating lever and treadle manipulanda or may be less willing than normal animals to engage in such behaviors.

Although it has been argued that the autoshaped response is not a conditional response (CR) in the traditional Pavlovian sense that it should be a product of the same efferent system as the unconditional response (UR) (Gormezano \& Kehoe, 1975), it does appear to follow Pavlovian associative rules (Hearst, 1979; Schwartz \& Gamzu, 1977). The autoshaping 
procedure thus presents an opportunity to test the sensory/motor deficit explanation of the operant data since neodecorticates do not seem to be impaired in their ability to associate a Pavlovian conditional stimulus (CS) with an unconditional stimulus (US). Specifically, if their disability in the instrumental freeoperant situation is in lever- or treadle-pressing itself, the neodecorticates should be equally retarded when producing similar responses in the autoshaping situation. If, on the other hand, their problem is not one of motor integration or sensory analysis, but of forming the particular response-reward associations required in the free-operant paradigm, then they should be able to produce lever or treadle responses as reliably as do normal animals when trained under autoshaping conditions. The present studies involve neodecorticated rats in two different autoshaping procedures, both of which involve a manipulable (lever) CS.

\section{EXPERIMENT 1}

This experiment was an investigation of appetitive autoshaping (sign-tracking) in normal and neodecorticated rats, using moving levers as the CSs. Both leverpresses and lever contacts were recorded as measures of sign-tracking, and food-tray-door movements were taken as a measure of goal-tracking (Boakes, 1977; Jenkins et al., 1978).

\section{Method}

Subjects and Surgery. The subjects were four neodecorticated and four normal rats (male, Hooded Lister) with a mean body weight of $364.8 \mathrm{~g}(\mathrm{SD} \pm 25.5 \mathrm{~g})$ prior to surgery. The neodecortications were produced in one stage by removing the pial blood supply from the surface of both hemispheres over the neocortex (Oakley, 1979c). The normal animals were sham-operated with two 5-mm-diam trephine holes over parietal cortex. All surgery was under pentobarbitone sodium (Sagatal) anesthesia, and all animals had 20-28 weeks of postoperative recovery.

Apparatus. The experimental chamber was a standard operant box (Campden Instruments Ltd.), measuring internally $23.5 \times$ $23.0 \times 20.5 \mathrm{~cm}$ high, in a sound-attenuating enclosure. The box had two retractable levers on one end wall, $3.0 \mathrm{~cm}$ to the left and right of a food tray into which Noyes reinforcement pellets $(45 \mathrm{mg}$ ) could be delivered. When extended, the levers projected $2.2 \mathrm{~cm}$ into the box, were $1.0 \mathrm{~cm}$ thick and $3.8 \mathrm{~cm}$ wide, and were located $4.0 \mathrm{~cm}$ above the grid floor. When retracted, the levers were flush with the wall. When extended, a pressure on the top of the lever equivalent to a weight of $8 \mathrm{~g}$ was required to operate it and was recorded as a press. Lever contacts of insufficient force to cause a press were recorded by drinkometer circuits. The food-tray aperture was $5.0 \mathrm{~cm}$ high $\times 4.0 \mathrm{~cm}$ wide, with a tophinged transparent Perspex door to record tray entries. Foodpellet delivery was accompanied by the noise of the dispenser and a brief flash of light in the food tray. General illumination was by a small houselight on the ceiling of the box. Two identical operant boxes were used simultaneously to test the animals.

Body weight reduction and magazine training. In the two groups of animals used here, the normals could maintain their body weights on $15 \mathrm{~g}$ of food per day, while the neodecorticates, despite their generally lower mean body weights, required $20 \mathrm{~g}$. Body weight reduction to $85 \%$ of its free-feeding value was achieved by feeding slightly less than these amounts per day. The neodecorticates reached the desired level in 18 days, whereas an additional 2-6 days were required for the normal animals. The greater sensitivity of neodecorticates to food deprivation has been noted previously, as had their lower set-point for body weights under freefeeding conditions (Braun, 1975; Oakley, 1971, 1979b, 1980; Oakley \& Russell, 1975, 1976, 1978b; Vanderwolf, Kolb, \& Cooley, 1978). Each animal was then placed in an operant box, with both levers retracted, for 21 daily sessions of magazine training. During each session, food pellets were delivered every $60 \mathrm{sec}$ until 30 pellets had been presented. At the beginning of the first few sessions, five additional pellets were placed in the food tray.

Autoshaping: Single-lever acquisition. For the following 10 sessions, food delivery (US) was paired with the insertion of one lever (CS) into the chamber. The left lever (LL) in all subjects was inserted into the chamber for $10 \mathrm{sec}$ prior to food delivery and was retracted as the food pellet was dispensed. These CS-US presentations occurred on a variable time schedule of $100 \mathrm{sec}$ (VT100). The right (RL) was never inserted during these acquisition sessions. Each session lasted for $\mathbf{4 0}$ food deliveries. At no stage of autoshaping did lever contacts or presses have any effect on the delivery of food pellets until the introduction of omission schedules in Experiment 3.

Autoshaping: Differentiation and reversal. During differentiation training (10 sessions), LL remained the positive CS $(\mathrm{CS}+)$ and continued to reliably predict the delivery of food. The RL was also inserted into the chamber for $10-\mathrm{sec}$ periods, but on a separate VT100 schedule and independently of food. RL thus predicted food only on a chance basis $\left(\mathrm{CS}^{\circ}\right)$. For an additional 40 sessions, the significance of $L L$ and $R L$ insertions was reversed so that RL became CS + and LL became CS $^{\circ}$.

Histology. When testing was complete, the animals were given a lethal dose of pentobarbitone sodium (Sagatal). followed by an intracardiac perfusion of physiological saline, and then a $10 \%$ formol saline solution. Brains were removed, drawn, photographed, and embedded in paraffin wax. Coronal sections were cut at $15 \mu$ thickness, and every 17 th section was stained with cresyl violet and mounted under glass. The lesions were drawn from the prepared sections onto a series of standard coronal sections and were then reconstructed individually onto an outline drawing of dorsal and lateral aspects of a normal rat brain. Estimates of lesion size were obtained by perimetry from the series of coronal sections.

Statistical analyses. In all three experiments, between-groups analyses were based on Mann-Whitney U tests (Siegel, 1956), and within-group trends were evaluated using Page's L test (Page, 1963). Related means $t$ tests were used in Experiment 3 for data derived from testing the same animal under two conditions.

\section{Results}

Histology. Since lesion sizes and distributions were very similar across experiments and to those reported previously (see, e.g., Oakley 1979c), combined histological data, based on a sample of seven neodecorticates drawn from Experiments 1 and 2, are presented here. The percentage of total neocortex removed ranged from $92.31 \%$ to $100.00 \%$ (mean $98.70 \mathrm{SD} \pm$ 2.84). Figure 1 is a composite surface reconstruction of all seven brains, showing the area common to all lesions (hatched) and the range of lesion variability (dotted). The small amount of neocortical sparing in these brains was predominantly anterior, adjacent to the rhinal fissure. Transitional cortex was damaged in the majority of brains along the rhinal fissure, particularly caudally, and in the midline. Postoperative necrosis had removed all devascularized cortical 

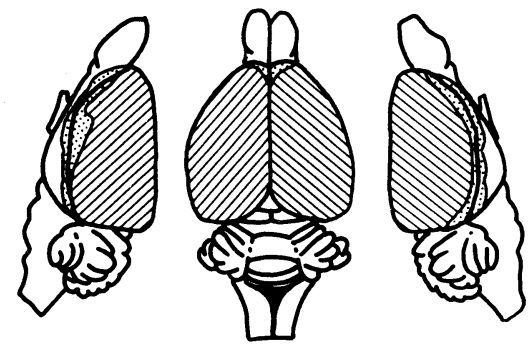

Figure 1. Dorsal and lateral surface reconstructions of neocortical lesions in seven animals from Experiments 1 and 2, showing tissue loss common to all animals (hatched) and the range of lesion boundaries (dotted).

tissue, and, in most instances, the majority of associated white matter, including the corpus callosum, had also disappeared. Ventricular expansion had occurred in all the lesioned brains, causing displacement of caudate-putamen. Hippocampus was less frequently displaced, but, in Animal U16 from Experiment 2 , slight scarring of dorsal hippocampus was evident in the right hemisphere. No other evidence of subcortical damage was seen in any brain. Careful analysis revealed no correlation between performance and lesion size, lesion shape, or the presence of subcortical damage or distortion in either experiment.

Postoperative recovery and magazine training. All four neodecorticates involved in Experiment 1 were maintaining their own stable body weights on standard laboratory diets and plain water from drinking bottles at the time the experiment commenced. No obvious behavioral abnormalities were present on gross observation in the home cage or in the general laboratory setting. There were no significant differences in ease of magazine training as measured by tray entries per session between normal and neodecorticate groups. Only one animal, neodecorticate No. 24, required tray-door shaping in the form of the tray door's being held half open and progressively lowered to its normal resting position over a period of five sessions.

Autoshaping. Figure 2 summarizes mean lever contacts per CS + and $\mathrm{CS}^{0}$ trial for both groups during acquisition, differentiation, and reversal stages of training. Both groups show increasing levels of contact with CS + (LL) during acquisition, although this trend is not significant in either group, and there is no significant difference in CS + contact levels between the two groups ( $p>.05$ in all cases). With the introduction of differentiation, clear differences in response distribution between $\mathrm{CS}+$ and $\mathrm{CS}^{\circ}$ were evident in both groups. Calculating differentiation scores as (Responses to CS +)/(Responses to CS + and $\mathrm{CS}^{\circ}$ ) gives mean values for contacts over all 10 differentiation sessions of $.925(\mathrm{SD} \pm .033)$ for normals and $.903(\mathrm{SD} \pm .067)$ for neodecorticates.
There were no between-groups differences in differentiation scores or CS + contacts during differentiation. Under reversal conditions, both groups were able to reverse the distribution of contacts between levers, although this reversal was significantly more complete in the lesioned group $(p=.029)$. Mean differentiation scores on the final four sessions of reversal were $.589(\mathrm{SD} \pm .237)$ for the normal group and $.8(\mathrm{SD} \pm .052)$ for the neodecorticates.

Trends for leverpresses were the same as those described for contacts in both groups, although, interestingly, presses account for $62.0 \%(\mathrm{SD} \pm 17.5 \%)$ of contacts in the neodecorticate group, whereas a significantly smaller proportion, $12.7 \%(\mathrm{SD} \pm 12.1 \%)$, of contacts result in presses in the normal group $(\mathrm{p}=.014)$.

Mean numbers of tray entries per CS trial for CS + and $\mathrm{CS}^{\circ}$ are shown for both groups in Figure 3. Also shown for the acquisition stage is the baseline level of tray investigation, calculated as the mean number of food-tray-door responses per $10 \mathrm{sec}$ when no CS is present. Baseline tray-door activity was significantly higher in the neodecorticates $(p=.029)$, but did not change from its acquisition levels in either group. Levels of tray entry (TE) during CS + were not significantly different in the two groups under acquisition, differentiation, or reversal conditions. Calculating differentiation scores as before, but using the

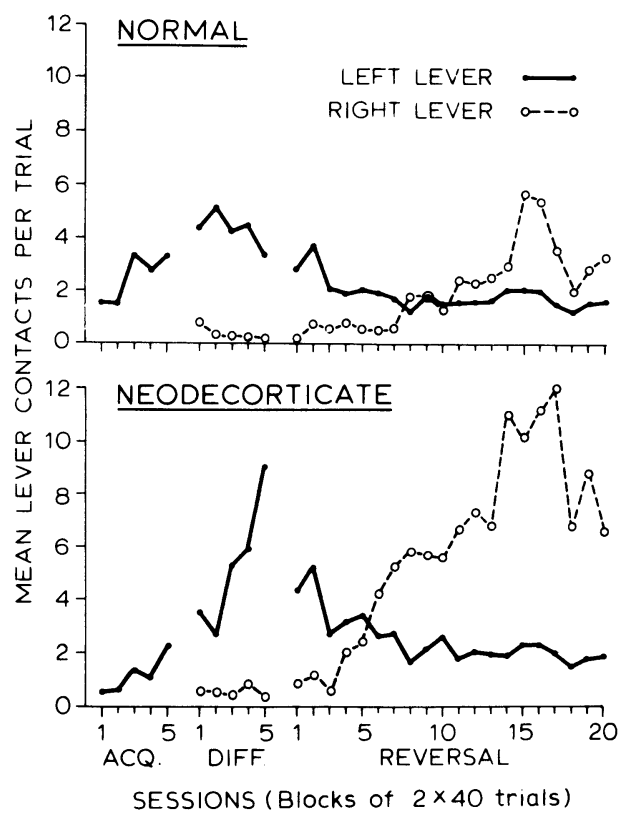

Figure 2. Mean number of lever contacts during each CS presentation in normal and neodecorticated rats during acquisition (ACQ), differentiation (DIFF), and reversal stages of movinglever autoshaping, plotted in two session blocks. Left lever was CS + during acquisition and differentiation, and right lever was $\mathrm{CS}+$ during reversal. 


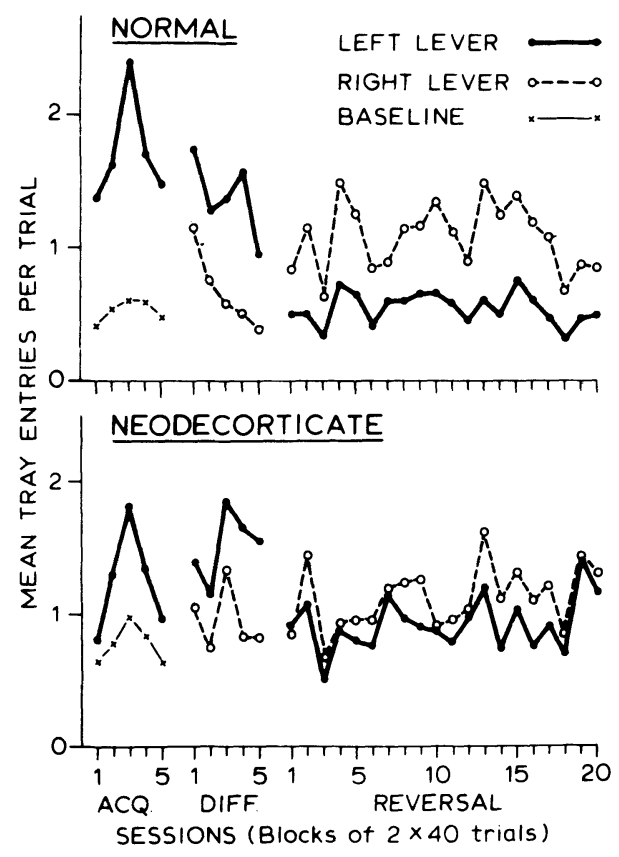

Figure 3. Mean number of tray-door responses during each CS (lever) presentation and per 10 -sec period outside CS presentation (baseline) in normal and neocorticated rats during acquisition (ACQ), differentiation (DIFF), and reversal stages of movinglever autoshaping, plotted in two-session blocks. Left lever was $\mathrm{CS}+$ during acquisition and differentiation, and right lever was $\mathrm{CS}+$ during reversal. Baseline data are shown for acquisition only.

baseline measure instead of $\mathrm{CS}^{\circ}$ levels, however, reveals differential TE scores during acquisition of .776 $(\mathrm{SD} \pm .017)$ for normals and .631 (SD \pm .025$)$ for neodecorticates, which is a significant difference $(p=.014)$, reflecting a greater tendency on the part of normals to make TEs in response to CS + than at other times. Differentiation scores based on TEs during CS + and $\mathrm{CS}^{\circ}$ were similarly higher in normals than in neodecorticates during differentiation [normal .661 (SD \pm .088 ); neodecorticate $.607(\mathrm{SD} \pm .057)$ ] and reversal [normal .625 (SD \pm .123$)$; neodecorticate .517 (SD \pm .039$)$ ], although in neither case was the between-groups difference significant.

Levels of sign-tracking (lever contacts) and goaltracking (TEs) during CS + are compared in Figure 4, individually for all four animals in both groups for the final four sessions of acquisition, differentiation, and reversal. This figure has been drawn with the animals in each section ranked in ascending order of lever contacts to $\mathrm{CS}+$. CS $+\mathrm{TE}$ levels vary relatively little within groups, are similar between groups, and show no consistent relationship with lever contact levels. There is marked variation, however, in levels of sign-tracking. At the end of acquisition, two normals (26 and 28 ) and two neodecorticates (23 and 24) had begun to sign-track. In the final four sessions of differentiation, all four animals in both groups were tracking $\mathrm{CS}+$ with contacts, with huge variation in levels of contact among the neodecorticates. At the end of reversal, three normals and all four neodecorticates were tracking the new CS + (RL) by contacting it, with this tendency being far more marked in the neodecorticates than in the normal rats.

\section{Discussion}

It seems clear that neodecorticated rats are at least as likely as normal animals to form signal-centered action patterns to the CS in an autoshaping situation. They are also as capable as normals of differentiating a predictive CS from a nonpredictive CS and of reversing that differentiation when the predictiveness of the two stimuli is reversed. Of particular interest here is the fact that, although there was no difference in the level of CS + contacts during acquisition, a far higher proportion of those contacts resulted in

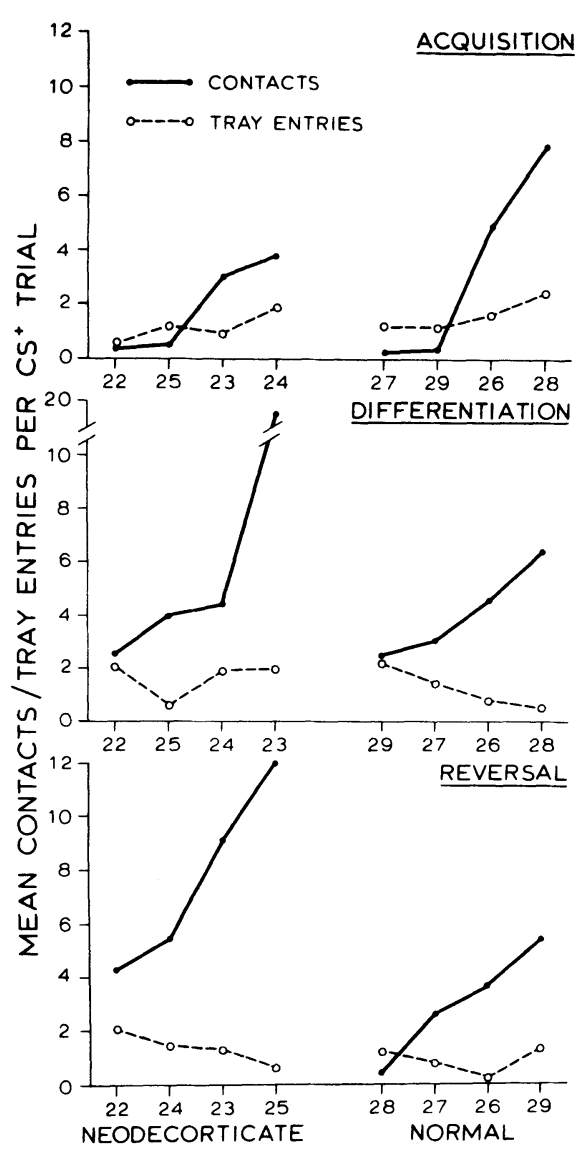

Figure 4. Mean number of lever contacts and food-tray responses during each CS + presentation for four neodecorticates (Animals 22-25) and four normals (Animals 26-29) in the final four sessions of acquisition, differentiation, and reversal training with moving levers. Each animal is identified beneath the horizontal axis in each part of the figure, and the data are arranged from left to right in ascending order of mean lever contacts. 
actual leverpresses in the neodecorticates than was the case in normals. The normal levels of differentiation and superior reversal performance of the neodecorticates on the contact measure of sign-tracking in this experiment is reminiscent of the performance of neodecorticates in a more traditional Pavlovian situation (Oakley \& Russell, 1974, 1975, 1976, 1977) and contrasts with the slower-than-normal response reversals seen in instrumental learning paradigms (Oakley, 1979b, 1981).

Tray entries during CS were as common in neodecorticates as in normals, although the normals showed a greater tendency, particularly in reversal, to make the response differentially to $\mathrm{CS}+$. The failure of differential goal-tracking under reversal conditions in the neodecorticates is in sharp contrast with the very clear differential sign-tracking that they demonstrated to the same CSs at this stage of training. This dichotomy suggests independent control of signtracking and goal-tracking by $\mathrm{CS}+$ and $\mathrm{CS}^{\circ}$ in the neodecorticates. Individual data analysis confirmed for both the neodecorticates and the normals the high within-groups variability previously reported for autoshaping in normal animals (Boakes, 1977; Jenkins et al., 1978) but failed to provide evidence in either group for the competitive relationship described by Boakes (1977) between sign-tracking and goal-tracking. Boakes, however, first described this relationship between CS- and US-oriented behaviors in studies in which a stationary illuminated lever was used as the CS. Our observations would seem to strengthen his tentative conclusion that moving lever CSs may minimize the conflict between sign-tracking and goal-tracking behaviors. A second experiment was conducted, using neodecorticated and normal rats, in which the CS was the illumination of a lever, in order to, first of all, establish the generality of the various observations made in Experiment 1 and, secondly, to evaluate sign-tracking and goal-tracking in a situation thought to enhance the conflict between them in normal animals.

\section{EXPERIMENT 2}

This experiment investigated differential appetitive autoshaping in normal and neodecorticated rats, using illuminated levers as the conditional stimuli and comparing sign-tracking and goal-tracking behaviors.

\footnotetext{
Method

Subjects and Surgery. The subjects were five normal and five neodecorticated rats (male, Hooded Lister) with a mean body weight of $353.9 \mathrm{~g}(\mathrm{SD} \pm 21.8 \mathrm{~g})$ prior to surgery. The neodecortications and sham operations were carried out as described in Experiment 1, and all animals had 16-17 weeks of postoperative recovery.
}

Apparatus. The apparatus was as in Experiment 1, except that two fixed levers that could be illuminated during CS presentation were used in place of retractable levers. The illuminable levers were $5.0 \mathrm{~cm}$ wide and $1.0 \mathrm{~cm}$ thick and protruded $1.3 \mathrm{~cm}$ into the box. They were of transparent Perspex and were covered on both sides, the top, and the bottom with stainless steel sheet. The front and rear edges of the lever had no metal cover and allowed the light from a 24-V, 2.8-W light bulb placed behind the lever to shine into the box. Lever contacts, presses, and traydoor movements were recorded as in Experiment 1.

Body weight reduction and magazine training. The body weights of all animals were reduced, as before, to approximately $85 \%$, and the greater sensitivity of the lesioned animals to food deprivation was again noted. Magazine training was also as described above, except that only 12 sessions were given and the first five of these consisted of tray-door shaping. This involved the tray door's being held open during the first session and then progressively lowered to its normal resting position by Session 6 .

Differential autoshaping. Magazine training was followed directly by differential autoshaping, in which the CSs consisted of a 10-sec-duration illumination of a lever followed, in the case of CS + only, by the delivery of a food pellet (US). For three animals in each group, illumination of the right lever $(R L)$ was the $\mathrm{CS}+$ and illumination of the left lever (LL) served as $\mathrm{CS}^{\circ}$. The predictiveness of the two levers with respect to the US was the reverse of this for the remaining two animals in each group. CS + and $\mathrm{CS}^{\circ}$ were controlled by independent VT120 schedules and were each presented 30 times per session. Food pellet delivery was signaled by the noise of the dispenser and the switching on of a small light inside the food tray. This light was switched off as soon as the animal opened the food-tray door.

\section{Results}

Histology. See Results section of Experiment 1 and Figure 1.

Postoperative recovery and magazine training. Postoperative recovery was as in Experiment 1, and no significant differences were found between groups in the time taken for successful magazine training.

Differential autoshaping. Mean numbers of contacts to CS + and $\mathrm{CS}^{\circ}$ during all 15 sessions of differential autoshaping are shown for both groups of animals in Figure 5, with data for the first session presented as five-trial blocks. The increasing tendency of neodecorticates to track CS + with contacts is evident within the first session and forms a significant trend $(p<.01)$ over all 15 sessions, as does their decreasing tendency to contact $\mathrm{CS}^{0}(\mathrm{p}<.05)$. These trends result in a mean differentiation score for contacts of $.892(\mathrm{SD} \pm .081)$. In marked contrast, the normal animals, as a group, showed no significant increase in CS + contacts over 15 sessions of training or at any point within that period. The mean number of CS + contacts produced during the final four sessions was significantly lower in the normal group compared with the neodecorticates $(p=.008)$. Apart from lower numerical values, the leverpress data were similar to those for contacts. The percentage of lever contacts that resulted in presses in the neodecorticates in this experiment was $23.03 \%$.

Figure 6 shows the mean numbers of food-tray entries (TEs) per CS + and $\mathrm{CS}^{\circ}$ presentation in both groups. TEs during CS + are higher, although not 


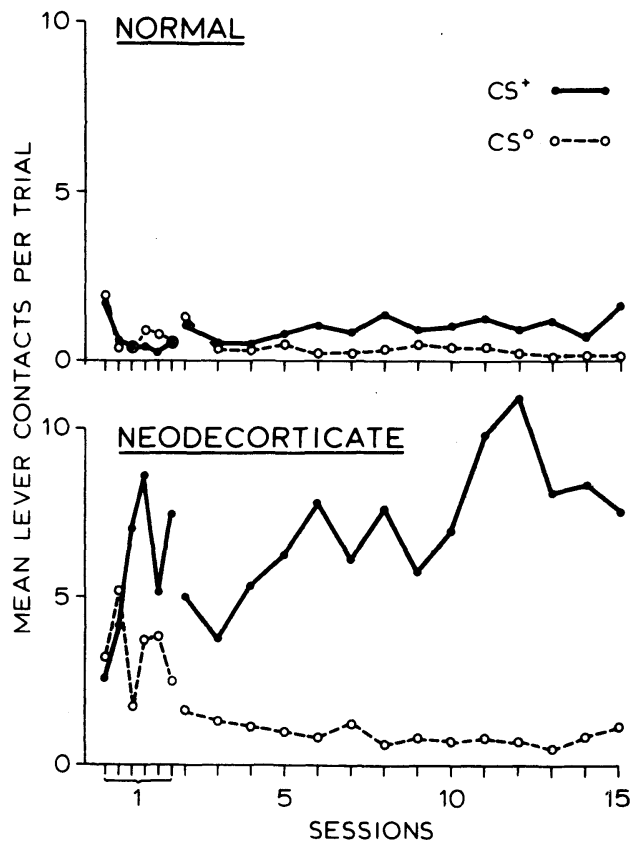

Figure 5. Mean number of lever contacts during each CS presentation in normal and neodecorticate rats during illuminatedlever autoshaping. Session 1 is shown in five-trial blocks and Sessions $2-15$ are plotted as whole (30-trial) sessions.

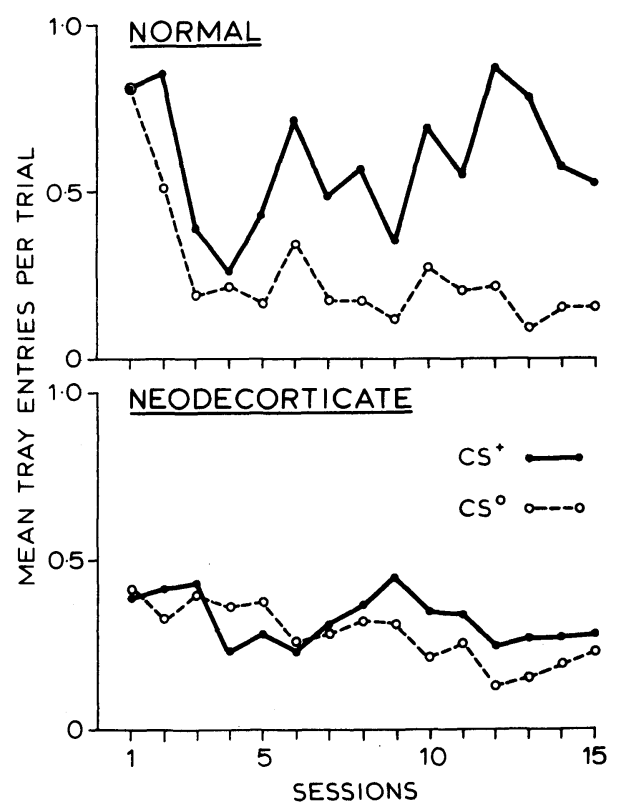

Figure 6. Mean number of tray-door responses during each CS (lever) presentation in normal and neodecorticated rats during illuminated-lever autoshaping.

significantly so, in normals, compared with neodecorticates. CS $^{0}$ levels are higher in the normals over the first two sessions, but drop to levels not significantly different from those produced by the lesioned an- imals thereafter. The differentiation between $\mathrm{CS}+$ and $\mathrm{CS}^{\circ}$ as signals for TE is, as in Experiment 1, more marked for the normal animals [differentiation score for the final four sessions $=.604(\mathrm{SD} \pm .224)]$ than it is for the neodecorticates [.478 $(\mathrm{SD} \pm .153)]$, although the difference is not significant.

Figure 7 gives mean numbers of lever contacts and TEs during CS + over the final four sessions of training as individual scores for all five animals in each group. As in Figure 4, the animals are ranked in order of increasing levels of CS + contact (signtracking), with goal-tracking represented by mean numbers of TEs per CS + trial. Taking TE scores of 1 or more per CS + trial as evidence of goal-tracking, three normal animals (U8, U9, U11) can be claimed as goal-trackers. Consistent with this categorization is the fact that these animals also show differential distributions of TEs during CS + and $\mathrm{CS}^{\circ}$ (TE differentiation scores for U8, U9, and U11 are .78, .74, and .78, respectively). The normals $\mathrm{U} 12$ and $\mathrm{U} 13$ would be classed as non-goal-trackers on the basis of absolute levels of TE and have TE differentiation scores of .34 and .38 , respectively. Only one of the five normal animals, U13, can be classed as a signtracker on the basis of its CS + contact score. In contrast, the neodecorticates present a simple picture characterized by a clear tendency to sign-track. Goal-tracking, on the other hand, is not present in the lesioned animals either in terms of absolute levels or as a differential distribution of TEs during CS + and $\mathrm{CS}^{\circ}$ (see Figure 6 and TE differentiation score for neodecorticates).

Concerning a possible competitive relationship between sign-tracking and goal-tracking, it is true that all the neodecorticates show a strong tendency toward sign-tracking and little evidence of goal-tracking. There is, however, no clear interaction between the level of contacts and of TEs for these animals (see Figure 7), and an analysis of individual acquisition data showed no suppression of goal-tracking by the emergence of sign-tracking or vice-versa. The normal animals do, however, provide some evidence of competition between the two behaviors. First, all the animals that were classed as goal-trackers (U8, U9, and U11) showed low levels of lever contacts, and, conversely, the one normal animal that sign-tracked (U13) showed no goal-tracking. Perhaps more convincing is that U13 had levels of goal-tracking in early sessions similar to those seen in U8, U9, and U11, but, from Session 7 onward, when it began to sign-track, tray entries during CS + dropped to zero.

Data collected for leverpresses between CS presentations showed that, despite a far lower absolute level of responding compared with that with $\mathrm{CS}+$ present, all the neodecorticates and the one normal animal that sign-tracked (U13) responded differentially to the two levers. Between-trial differentiation scores for presses on the CS + and CS $^{\circ}$ levers were 


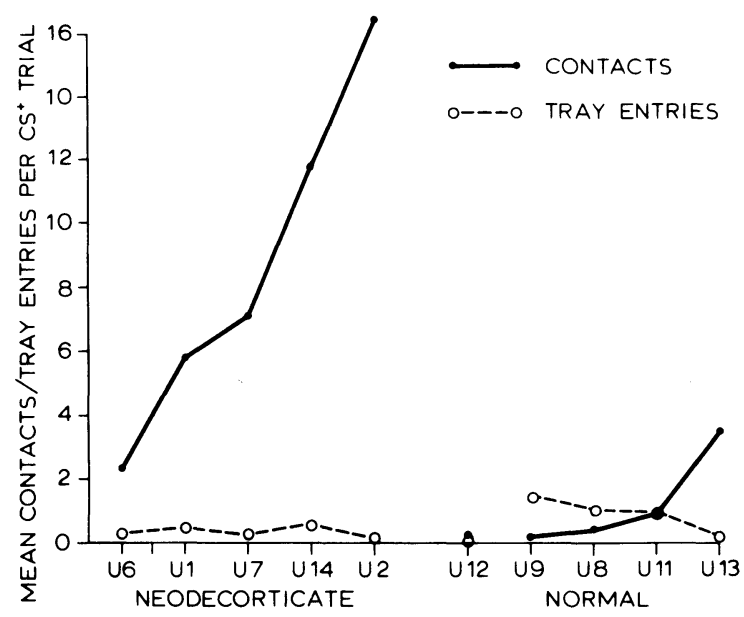

Figure 7. Mean number of lever contacts and tray-door responses during each CS + presentation for five neodecorticated and five normal animals on the final four sessions of illuminated lever autoshaping. The animals are identified by the prefix $U$ and a number beneath the horizontal axis and are arranged from left to right in each group in ascending order of mean lever contacts. U12 in the normal group is plotted separately, since it was the only animal to show neither contacts nor tray entries during $\mathbf{C S}+$.

$.766(\mathrm{SD} \pm .067)$ for the neodecorticates, .862 for $\mathrm{U13}$, and $.463(\mathrm{SD} \pm .163)$ for the normal animals that did not sign-track.

\section{Discussion}

With illuminated levers, the neodecorticates tracked $\mathrm{CS}+$ more readily than did normal animals and, once again, differentiated between a signal that predicted the US and one that did so only on a chance basis. In contrast with this clear evidence for signtracking, the neodecorticates did not show any tendency toward goal-tracking. That is, they used the illumination of the CS + lever as a signal to produce approach and manipulatory responses toward it but did not use the same CS + as a signal for US-oriented action patterns. The normals, as a group, however, were more prone to goal-tracking, and only one of the five animals in this group produced CS-oriented action patterns of sufficient intensity to be recorded as contacts or presses. The normal data are consistent with an analysis in terms of conflict between signtracking and goal-tracking behaviors and are comparable in variability to those described for normal rats by Boakes (1977) and to those described for dogs by Jenkins et al. (1978). The predominantly US-oriented action patterns in our normals may be related to the type of CS used. Boakes (1977) reported that sign-tracking to a visual CS in rats was enhanced if the lever was illuminated on its upper surface. Our lever was illuminated at the end, with little light falling on its upper surface. This is a rel- atively simple stimulus that contains little extra information that can be obtained by closer investigation, and so may encourage visual tracking at a distance and enhance US-oriented behaviors in normal animals. The same considerations evidently do not apply with equal force to neodecorticates, however, since they tracked the illuminated lever with physical contacts, although it is also true that these contacts were less vigorous than those elicited by a moving lever, insofar as a smaller proportion of them resulted in leverpresses.

It has been suggested (Oakley, 1979b, 1980; Oakley \& Russell, 1978a, 1978b, 1979, 1980) that one cause of inefficiency in neodecorticates in free-operant situations is a failure to identify the manipulandum as a discrete entity of particular behavioral significance. The neodecorticates in the present experiment continued to show differential response patterns to the two levers between CS trials and had apparently identified the levers on the basis of spatial and other cues, independently of their illumination. If the manipulandum identification hypothesis is correct, it would be predicted that neodecorticates given prior training in this or similar autoshaping situations would be able to progress to higher schedules of reinforcement and to perform as efficiently as normals in free-operant situations. Pilot studies just completed suggest that this is the case. Three neodecorticated rats pretrained in a moving-lever autoshaping paradigm were able to produce up to 35 leverpresses per reinforcement (which was the highest ratio tested) consistently and efficiently. This contrasts with an upper limit of 4-10 presses per reinforcement seen in naive neodecorticates (Oakley, 1971; Oakley \& Russell, 1978a, 1978b, 1979, 1980). By enhancing manipulandum identification, therefore, autoshaping may serve as an effective remedial pretraining procedure for subsequent free-operant performance involving the same manipulandum in neodecorticates.

\section{EXPERIMENT 3}

The neodecorticates in Experiments 1 and 2 showed higher than normal levels of autoshaped leverpresses and contacts. In many normal animals, particularly pigeons, powerful CS-directed responding may persist even if the execution of the autoshaped response serves to prevent delivery of the food US on that trial (Jenkins, 1973; Peden, Browne, \& Hearst, 1977; Williams \& Williams, 1969). Normal rats will suppress lever contacts but not eliminate them completely when lever presence predicts food but lever contacts prevent it (Atnip, 1977). The final experiment here involved an omission schedule to assess the persistence of the neodecorticate's autoshaped behavior when lever responses during the CS + serve to pre- 
vent food delivery. The introduction of an omission contingency, however, also reduces the frequency of reinforcement on the Pavlovian autoshaping schedule, and this in itself could reduce the level of leverpressing. To control for any partial reinforcement effect, a yoked control design was employed.

\section{Method}

Subjects and Apparatus. The subjects were the four neodecorticated rats from Experiment 1 divided into two pairs, with one of each pair designated the master (omission) animal, while the other served as the yoked control. The apparatus was the same two operant boxes used in Experiment 1. Each pair of animals was tested simultaneously, one in each box, and all animals were tested in the box in which they had been trained previously.

Omission and yoked training. The autoshaping procedure was the same as that at the end of Experiment 1, with the right lever as $\mathrm{CS}+$ and the left lever as $\mathrm{CS}^{\circ}$. If the master animal pressed the right lever during its insertion as $\mathrm{CS}+$, however, the food pellet (US) was not delivered on that trial to either the master of the yoked animal. Lever contacts by the master animal had no effect on food delivery, provided they did not result in a press, and no aspect of the yoked animals' behavior affected food delivery. After 20 sessions, the roles of the two animals were reversed and an additional 20 sessions of training were given. The normal animals, as a group, did not show differential autoshaping reliably at the end of Experiment 1 and so did not provide an adequate baseline for the omission procedure. Consequently, data were collected from only neodecorticates in this experiment.

\section{Results}

The data presented here are for leverpresses, since this was the particular response specified in the omission schedule, but the data for lever contacts are parallel in all respects. As the omission and yoked conditions were counterbalanced within the group, all the omission data and all the yoked control data were pooled for statistical analysis. The major comparisons are shown in Figure $\mathbf{8}$ for leverpresses and tray entries (TEs) during $\mathrm{CS}+$ and $\mathrm{CS}^{\circ}$ presentations during the first and final four sessions of training under omission and yoked control conditions. Leverpressing during CS + showed a significant downward trend over sessions in the omission condition $(p<.01)$ to reach a level on the final four sessions significantly below $\mathrm{CS}+$ levels at the end of training in the yoked condition $(p<.05)$ and not significantly different from $\mathrm{CS}^{\circ}$ response levels. Under yoked conditions, on the other hand, no significant trend appeared in either direction for numbers of presses per trial, and CS + responding remained significantly higher than $\mathrm{CS}^{0}$ leverpress levels $(\mathrm{p}<.05)$. TEs were not differentially altered by the omission and yoked conditions or by changes in leverpress levels and, as in the previous two experiments, were not differentially distributed between $\mathrm{CS}+$ and $\mathrm{CS}^{\circ}$.

\section{Discussion}

Removal of neocortex thus does not seem to prevent response suppression, at least to $\mathrm{CS}^{0}$ levels, when an omission schedule is superimposed on an autoshaped response baseline, despite the persistently
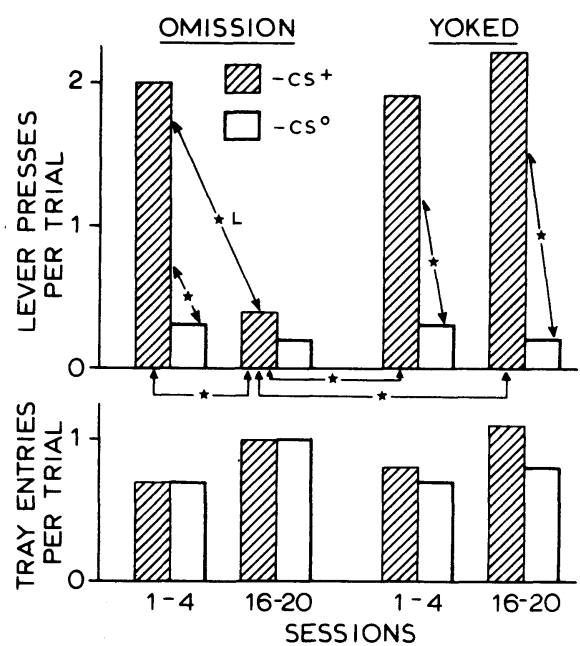

Figure 8. Mean number of leverpresses and tray entries per $\mathrm{CS}+$ and $\mathrm{CS}^{\circ}$ presentation for the first and final four sessions of moving-lever autoshaping under omission and yoked conditions in four neodecorticated rats. $L=$ Page's $L$ test for trend. ${ }^{*} \mathrm{p}<.05$

high levels of autoshaped responding seen in the neodecorticates. There is currently debate whether response suppression under these conditions is evidence of the formation of response-reinforcer associations or is a reflection of changes in stimulus-reinforcer relationships introduced by the omission contingency itself (Davey, Oakley, \& Cleland, 1981; Dickinson \& Mackintosh, 1978; Mackintosh \& Dickinson, 1979). The present observations could thus reflect once more the sensitivity of the neodecorticate to Pavlovian conditioning procedures and to changes in stimulusreinforcer relationships. On the other hand, they might be taken as evidence of the formation of an association between a leverpress and its environmental consequences. What is clear, however, is that, under appropriate conditions, the neodecorticate is able to withhold a well-established autoshaped response to $\mathrm{CS}+$ despite the fact that this stimulus continues to predict the delivery of the food US. This result is in general agreement with studies showing that neodecortication does not impair response inhibition in more traditional Pavlovian situations employing a conditioned inhibition design (Moore, Yeo, Oakley, \& Russell, 1980).

\section{GENERAL DISCUSSION}

These experiments, first of all, indicate that total removal of neocortex does not render rats either unable or unwilling to produce leverpresses when these responses are generated by a procedure governed by Pavlovian associative rules. The reverse in fact would seem to be the case, with higher levels of leverpressing in the lesioned animals than in the normal animals. It seems unlikely, therefore, that the neode- 
corticate rat's impairment in free-operant leverpressing situations is due to any fundamental difficulty in locating or operating the manipulandum. Second, it is generally accepted that autoshaping is controlled primarily by Pavlovian stimulus-reinforcer associations. As such, the results reported here confirm and extend earlier studies using more traditional Pavlovian procedures, which have shown that neodecorticates have no deficit in conditional response acquisition, that they are capable of perfect differentiation between predictive and nonpredictive CSs, that they display rather more rapid and complete reversals of that differentiation in comparison with normal animals (Oakley \& Russell, 1972, 1974, 1975, 1976, 1977), and that they have a normal capacity for response inhibition in a conditioned inhibition design (Moore et al., 1980). As far as the relationship between sign-tracking (CS-centered action patterns) and goal-tracking (US-centered action patterns) during the CS is concerned, our neodecorticates were signtrackers and showed little tendency to goal-trackespecially if differential responding to the US site during $\mathrm{CS}+$ and $\mathrm{CS}^{\circ}$ is taken as a criterion of goaltracking. It would seem, from the latter observation, that the intrusion of tray-door responses into instrumental response chains previously reported during the free-operant performance of neodecorticates (Oakley, 1979b, 1980; Oakley \& Russell, 1978a, $1978 \mathrm{~b}, 1979,1980)$ is not a specific case of a more general tendency to produce strong goal-tracking responses to predictive stimuli. The normal animals in these studies showed a generally greater tendency to goal-track, which in the second experiment took precedence over sign-tracking in most subjects.

The present differential autoshaping data suggest that the neodecorticates were able to distinguish adequately the illumination or movement of the CS + lever as well as the visual, auditory, and olfactory cues that accompanied food delivery. It could be, however, that they had difficulty in distinguishing the unilluminated food tray during CS presentations and that this might account for their reduced tendency to goal-track. Arguing against this interpretation, however, is the fact that the neodecorticates did enter the food tray prior to its illumination during CS + presentations at levels that, in the first study, were not significantly lower than normal. The neodecorticates were also more prone to look in the tray during the intertrial interval and during $\mathrm{CS}^{\circ}$ than were the normal animals. Similarly, there is evidence from earlier studies that, in addition to a greater-than-normal tendency to make tray exploration responses during operant responding on a lever, neodecorticates are as capable as normals of using an illuminated tray door as the manipulandum on fixed ratio schedules (Oakley, 1980). It is perhaps also worth mentioning at this point that the form of the autoshaped response may differ in the normal and lesioned animals. The neodecorticates were fre- quently seen to be "scrabbling" vigorously at the lever with both paws in a manner reminiscent of the way in which they retrieved food from the food tray. Normal animals in this type of autoshaping situation contact the lever predominantly with a single paw, with occasional nosing, licking, and biting responses consistent with the discrete motor actions associated with their food-retrieval behavior (Davey et al., 1981). While such differences have important theoretical implications, further consideration must await more systematic collection of the appropriate data on response topographies in the two groups.

In autoshaping, as in all formal learning procedures, it is assumed that some adaptive function is being tapped (see Jenkins et al., 1978; Plotkin \& Odling-Smee, 1979). In that case, since neodecorticates sign-track more reliably than do normal animals, are they showing greater amounts of an adaptive behavior or too much of a behavior that is only adaptive in smaller amounts? A number of considerations point to the latter conclusion. First, on a priori grounds, damaged brains would be expected to show less overall adaptiveness than intact brains, although this would clearly not preclude normal or even supranormal performance on particular tests, especially when these involve the testing of a simple function in isolation from other behaviors. High levels of sign-tracking on this basis must reflect some aspect of this reduced adaptiveness, and similar effects caused by neocortical lesions in other species have commonly been regarded as pathological. Stepien (1974), for example, has noted that prefrontal cortical lesions in dogs produce a "magnet" reaction whereby animals are drawn obsessively to the site of a CS predicting food. Interestingly, in view of our Experiment 2, the normal dogs in Stepien's study goal-tracked. Persisting in the investigation and manipulation of a predictive stimulus beyond the point at which no further information can be gained from so doing is also somewhat similar to the timeand energy-consuming hypercuriosity seen in dogs after early sensory restriction (Thompson \& Heron, 1954). There is also the point that sign-tracking can be maladaptive even in normal animals, particularly when goal-tracking would be the more appropriate behavior. The classic example of this is in the phenomenon of negative automaintenance, already described in connection with omission schedules (Williams \& Williams, 1969), but, even in the present studies, sign-tracking does not seem to be a particularly adaptive response insofar as it involves the expenditure of considerable energy for no material advantage. It is perhaps not so surprising, therefore, that normal animals show less sign-tracking and more of the possibly more appropriate behavior of goal-tracking. It is also true, however, that sign-tracking in the first two experiments described here did not have any particular disadvantage as far as obtaining the food reinforcer was concerned. Moreover, in the third ex- 
periment, in which they would have lost food pellets if they had persisted in tracking CS + under omission conditions, the neodecorticates ceased sign-tracking with both contacts and presses. While neodecorticates may show sign-tracking in excess of its immediate adaptiveness, they are thus apparently still capable of inhibiting, modifying, or redirecting their autoshaped responses when the behavior becomes positively disadvantageous.

\section{REFERENCES}

Atnip, G. W. Stimulus- and response-reinforcer contingencies in autoshaping, operant, classical and omission training procedures in rats. Journal of the Experimental Analysis of Behavior, 1977, 28, 59-69.

Boakes, R. A. Performance on learning to associate a stimulus with positive reinforcement. In H. Davis \& H. M. B. Hurwitz (Eds.), Operant-Pavlovian interactions. Hillsdale, N.J: Erlbaum, 1977.

BraUn, J. J. Neocortex and feeding behavior in the rat. Journal of Comparative and Physiological Psychology, 1975, 89, 507-522.

Davey, G. C. L., OAKley, D. A., \& Cleland, G. C. Effect of an omission contingency on the topography of autoshaped lever contacts and the relationship between sign-tracking and goal-tracking in the rat. Journal of the Experimental Analysis of Behavior, 1981, in press.

DiCara, L. V., Braun, J. J., \& Pappas, B. A. Classical conditioning and instrumental learning of cardiac and gastrointestinal responses following removal of neocortex in the rat. Journal of Comparative and Physiological Psychology, 1970, 73, 208-216.

Dickinson, A., \& Mackintosh, N. J. Classical conditioning in animals. Annual Review of Psychology, 1978, 29, 587-612.

Gormezano, I., \& Kenoe, E. J. Classical conditioning: Some methodological-conceptual issues. In W. K. Estes (Ed.), Handbook of learning and cognitive processes (Vol. 2). Hillsdale, N.J: Erlbaum, 1975.

Hearst, E. Classical conditioning as the formation of interstimulus associations: Stimulus substitution, parasitic reinforcement and autoshaping. In A. Dickinson \& R. A. Boakes (Eds.), Mechanisms of learning and motivation. Hillsdale, N.J: Erlbaum, 1979.

Hearst, E., \& Jenkins, H. M. Sign-tracking: The stimulusreinforcer relation and directed action. Austin, Tex: Psychonomic Society, 1974.

JENKINS, H. M. Effects of the stimulus-reinforcer relation on selected and unselected responses. In R. A. Hinde \& J. S. Hinde (Eds.), Constraints on learning. New York: Academic Press, 1973.

Jenkins, H. M., Barrera, F. J., Ireland, C., \& Woodside, B. Signal-centered action patterns in dogs in appetitive classical conditioning. Learning and Motivation, 1978, 9, 272-296.

Mackintosh, N. J., \& Dickinson, A. Instrumental (Type II) conditioning. In A. Dickinson \& R. A. Boakes (Eds.), Mechanisms of learning and motivation. Hillsdale, N.J: Erlbaum, 1979.

Moore, J. W., Yeo, C. H., OAKley, D. A., \& Russell, I. S. Conditioned inhibition of the nictitating membrane response in decorticate rabbits. Behavioural Brain Research, 1980, 1, 397-409.

OAKLEY, D. A. Instrumental learning in neodecorticate rabbits. Nature (New Biology), 1971, 233, 185-187.

OAKLEY, D. A. Cerebral cortex and adaptive behaviour. In D. A. Oakley \& H. C. Plotkin (Eds.), Brain, behaviour and evolution. London: Methuen, 1979. (a)

OAKLEY, D. A. Instrumental reversal learning and subsequent fixed ratio performance on simple and go/no-go schedules in neodecorticate rabbits. Physiological Psychology, 1979, 7, 29-42. (b)

OAKLEY, D. A. Learning with food reward and shock avoidance in neodecorticate rats. Experimental Neurology, 1979, 63, 627-642. (c)
OAKLEy, D. A. Neocortex and learning. Trends in NeuroSciences, 1979, 2, 149-152. (d)

OAKLEY, D. A. Improved instrumental learning in neodecorticate rats. Physiology \& Behavior, 1980, 24, 357-366. (a)

OAKLeY, D. A. Performance of neodecorticated rats in a twochoice visual discrimination apparatus. Behavioural Brain Research, 1981, in press.

OAkley, D. A., \& Russell, I. S. Neocortical lesions and Pavlovian conditioning. Physiology \& Behavior, 1972, 8, 915-926.

OAKLey, D. A., \& Russell, I. S. Differential and reversal conditioning in partially neodecorticate rabbits. Physiology \& Behavior, 1974, 13, 221-230.

OAKLey, D. A., \& Russell, I. S. Role of cortex in Pavlovian discrimination learning. Physiology \& Behavior, 1975, 15, 315-321.

OAkley, D. A., \& Russell, I. S. Subcortical nature of Pavlovian differentiation in the rabbit. Physiology \& Behavior, 1976, 17, 947-954.

OAKLey, D. A., \& Russell, I. S. Subcortical storage of Pavlovian conditioning in the rabbit. Physiology \& Behavior, 1977, 18, 931-937.

OAKLey, D. A., \& Russell, I. S. Manipulandum identification in operant behavior in neodecorticate rabbits. Physiology \& Behavior, 1978, 21, 943-950. (a)

OAkley, D. A., \& Russell, I. S. Performance of neodecorticated rabbits in a free-operant situation. Physiology \& Behavior, 1978, 20, 157-170. (b)

OAKLeY, D. A., \& Russell, I. S. Instrumental learning on fixed ratio and go-no go schedules in neodecorticate rats. Brain $\mathbf{R e}$ search, 1979, 161, 356-360.

OAKLey, D. A., \& Russell, I. S. Effect of prior experience on bar-pressing in rats without neocortex. Behavioural Brain Research, 1980, 1, 267-283.

Page, E. B. Ordered hypotheses for multiple treatments: A significance test for linear ranks. Journal of the American Statistical Association, 1963, 58, 216-230.

Peden, B. F., Browne, M. P., \& Hearst, E. Persistent approaches to a signal for food despite food omission for approaching. Journal of Experimental Psychology: Animal Behavior Processes, 1977, 3, 377-399.

Plotkin, H. C., \& Odling-Smee, F. J. Learning, change and evolution: An enquiry into the teleonomy of learning. Advances in the Study of Behavior, 1979, 10, 1-41.

Russell, I. S. Animal learning and memory. In D. Richter (Ed.), Aspects of learning and memory. London: Heinemann, 1966.

Russell, I. S. Neurological basis of complex learning. British Medical Bulletin, 1971, 27, 278-285.

Schwartz, B., \& Gamzu, E. Pavlovian control of operant behavior: An analysis of autoshaping and its implications for operant conditioning. In W. K. Honig \& J. E. R. Staddon (Eds.), Handbook of operant behavior. Englewood Cliffs, N.J: PrenticeHall, 1977.

SiEgEL, S. Nonparametric statistics for the behavioral sciences. New York: McGraw-Hill, 1956.

Stepien, I. The magnet reaction, a symptom of prefrontal ablation. Acta Neurobiologiae Experimentalis, 1974, 34, 145-160.

Thом pson, W. R., \& HERON, W. The effects of early restriction on activity in dogs. Journal of Comparative and Physiological Psychology, 1954, 47, 77-82.

Vande rwolf, C. H., Kolb, B., \& Cooley, R. K. Behavior of the rat after removal of the neocortex and hippocampal formation. Journal of Comparative and Physiological Psychology, 1978, 92, 156-175.

Williams, D. R., \& Williams, H. Automaintenance in the pigeon: Sustained pecking despite contingent non-reinforcement. Journal of the Experimental Analysis of Behavior, 1969, 12, 511-520.

(Received for publication September 30, 1980; revision accepted January 21,1981 .) 